Arinaldo VAsConcelos de Alencar ${ }^{1}$

FREDERICO TeIXERA BRANDT ${ }^{2}$

Hélo de Lima Ferrerira Fernandes Costa'

Carla Daisy Albuquerque ${ }^{3}$

RosânGela Falcão da Costa ${ }^{4}$

Saulo Barbosa Vasconcelos de Alencar ${ }^{5}$

Artigo original

Palavras-chave

Incontinência urinária por estresse/cirurgia Incontinência urinária por estresse/

ultrassonografia

Uretra/fisiopatologia

Bexiga urniária/fisiopatologia

Períneo/ultrassonografia

Keywords

Urinary incontinence, stress/surgery Urinary incontinence, stress/ ultrasonography

Urethra/physiopathology

Urinary bladder/physiopathology Perineum/ ultrasonography

\section{Modificações topográficas da junção uretrovesical e da uretra proximal após cirurgia combinada de Marshall-Marchetti- Krantz e Burch}

\author{
Topographic modifications of the urethrovesical junction and \\ proximal uretbra after combined Marshall-Marchetti-Krantz \\ and Burch surgery
}

\section{Resumo}

OBJETIVO: estudar, por meio da ultrassonografia perineal, as modificações da junção uretrovesical (UUV) e da uretra proximal (UP) promovidas pela cirurgia combinada de Marshall-Marchetti-Krantz-Burch (MMK-B). MÉTODOS: foi realizado um estudo de intervenção longitudinal e prospectivo. Trinta e duas mulheres com incontinência urinária de esforço foram submetidas à ultrassonografia perineal antes e 30 dias depois da cirurgia para avaliar a distância pubouretral (DPU), o comprimento da uretra proximal (UP), a distância horizontal da JUV (DHJUV) e a distância vertical da JUV (DVJUV), estando a paciente em repouso, e os seus deslocamentos durante a manobra de Valsalva. Os resultados foram expressos em médias e desvios padrão. Para a comparação dos resultados do pré aos do pós-operatório, foi usado o teste $t$ de Student para amostra pareada quando as variáveis cumpriam os critérios do teste de normalidade, e o teste pareado de Wilcoxon quando não cumpriam. RESULTADOS: em comparação às medidas do pré-operatório, a cirurgia de Marshall-Marchetti-Krantz-Burch reduziu a DPU no repouso (13,4 mm x 10,2 mm) e no esforço (19,8 mm × 9 mm); reduziu a DHJUV no repouso ( $14 \mathrm{~mm} \times 4,3 \mathrm{~mm}$ ) e no esforço (20,8 mm × 6,4 mm); aumentou o comprimento da UP no repouso (16,7 mm x 19,7 mm) e no esforço (1,6 mm x 15,4 mm); aumentou a DVJUV no esforço (-5,4 mm x $14,8 \mathrm{~mm}$ ). A cirurgia de MMK-B não alterou a DVJUV no repouso (16,2 mm x 18,7mm, p=0,085). CONCLUSÕES: a cirurgia de Marshall-Marchetti-Krantz-Burch reduziu significativamente a mobilidade vertical e horizontal da junção uretrovesical, porém sem elevar a junção uretrovesical.

\section{Abstract}

PURPOSE: to study the changes in the urethrovesical junction (UVI) and in the proximal urethra (PU) caused by the MarshallMarchetti-Krantz-Burch (MMK-B) combined surgery through perineal ultrasonography. METHODS: an interventional, longitudinal and prospective study has been conducted. Thirty-two women with stress urinary incontinence were submitted to perineal ultrasonography before and 30 days after surgery to evaluate the pubo-urethral distance (PUD), the proximal urethra length, the UVJ horizontal distance (UVJHD) and the UVJ vertical distance (UVJVD), the patient being at rest, and in effort during the Valsava manoeuvre. Results have been expressed in mean and standard deviation. The Student's t-test has been used to compare pre and postoperative results whenever the variables fulfilled the normality test criterion; otherwise, the Wilcoxon's paired test has been used. RESULTS: as compared with the preoperative measures, the Marshall-Marchetti-Krantz-Burch surgery has reduced the PUD at rest $(14 \mathrm{~mm} \times 4.3 \mathrm{~mm})$ and during effort $(20.8 \mathrm{~mm}$ $\times 6.4 \mathrm{~mm})$; has reduced the UVJHD at rest $(14 \mathrm{~mm} \times 4.3 \mathrm{~mm})$ and during effort $(20.8 \mathrm{~mm} \times 6.4 \mathrm{~mm})$; has increased the PU length at rest $(16.7 \mathrm{~mm} \times 19.7 \mathrm{~mm})$ and during effort $(1.6 \mathrm{~mm} \times 15.4 \mathrm{~mm})$; and has increased UVJVD during effort (-5.4 mm $\times 14.8 \mathrm{~mm}$ ), but has not changed it at rest (16.2 mm $18.7 \mathrm{~mm}, \mathrm{p}=0.085)$. CONCLUSIONS: the Marshall-Marchetti-Krantz-Burch surgery has significantly reduced the urethrovesical junction vertical and horizontal mobility without raising the urethrovesical junction.
Correspondência:

Arinaldo Vasconcelos de Alencer Avenida Beira Rio, 879 / 1.601 - Madaleno (EP 50610-100 - Recife (PE), Brasil

Fone: (81) 3226-4301

E-mail: arinaldoalencar@uol.com.br

Recebido

$13 / 8 / 08$

Aceito com modificacões
Unidade de Pesquisa em Incontinência Urinária da Universidade Federal de Pernambuco - UPIU-UFPE - Recife (PE), Brasil; Centro Integrado de Saúde Amaury de Medeiros da Universidade de Pernambuco - CISAM-UPE - Recife (PE), Brasil.

' Professores adjuntos da Disciplina de Tocoginecologia da Faculdade de Ciências Médicas da Universidade de Pernambuco - UPE - Recife (PE), Brasil.

2 Professor-associado da Disciplina de Urologia do Departamento de Cirurgia do Centro de Ciências da Saúde da Universidade de Pernambuco - UFPE - Recife (PE), Brasil.

${ }_{3}^{3}$ Professora adjunta da Disciplina de Urologia do Departamento de Cirurgia do Centro de Ciências da Saúde da Universidade de Pernambuco - UFPE - Recife (PE), Brasil.

${ }^{4}$ Mestranda em Cirurgia do Centro de Ciências da Saúde pela Universidade de Pernambuco - UFPE - Recife (PE), Brasil.

${ }^{5}$ Médico residente do Hospital das Clínicas da Universidade de Pernambuco - UFPE - Recife (PE), Brasil. 


\section{Introdução}

Caracterizada pela perda de urina pela uretra, sincrônica ao esforço físico, a incontinência urinária de esforço (IUE) ${ }^{1}$ é uma das formas predominantes de incontinência urinária na mulher, ocorrendo em 4,8 a $58,4 \%$ das mulheres ${ }^{2}$.

O principal fator determinante da IUE parece ser a descida exagerada da junção uretrovesical durante o esforço físico, sendo o parto a mais provável causa ambiental ${ }^{3}$. O pobre suporte anatômico do colo vesical, base da bexiga e uretra proximal resulta em hipermobilidade e descida da JUV e uretra proximal para fora da zona de transmissão da pressão abdominal para a uretra proximal, impossibilitando o aumento da pressão uretral para antagonizar a elevação da pressão vesical, favorecendo o surgimento da IUÉ . Com base nessas alterações, vem crescendo a importância da ultrassonografia transvulvar para o diagnóstico da IUE, pois, de maneira segura e reproduzível, evidencia a hipermobilidade da JUV, importante fator determinante da perda urinária ${ }^{4,5}$. O deslocamento vertical da JUV foi superior a $10 \mathrm{~mm}$ em $97 \%$ das 271 portadoras de IUE, e a sua constatação pela ultrassonografia perineal é de grande valor propedêutico ${ }^{4}$. Diferentes abordagens na avaliação ultrassonog ráfica da JUV são descritas ${ }^{6}$ e alguns autores consideram hipermobilidade da JUV quando o seu deslocamento vertical é maior que $9 \mathrm{~mm}^{7}$; outros o consideram quando esse deslocamento é maior ou igual a $10 \mathrm{~mm}^{4,8}$ e há aqueles que consideram hipermobilidade quando a resultante do deslocamento vertical e horizontal da JUV é maior que $15 \mathrm{~mm}^{9}$.

A aferição da altura e mobilidade da JUV é critério fundamental para orientar a forma de tratamento, o qual pode ser clínico e/ou cirúrgico, considerando que a cirurgia tem como finalidade impedir a hipermobilidade da JUV e uretra proximal ${ }^{9}$. Várias são as técnicas cirúrgicas usadas para tratar a IUE, destacando-se as cirurgias de Marshall-Marchetti-Krantz (MMK), Burch (B), e slings retropúbico e transobturador. $\mathrm{O}$ percentual de recidivas com o uso desses procedimentos aumenta com o tempo e, aos cinco anos, chega a $38 \%$ para a colpoplastia anterior e aproximadamente $21 \%$ para a cirurgia de Burch e sling retropúbico ${ }^{10,11}$. A cirurgia combinada de MarshallMarchetti-Krantz e Burch (MMK-B) levou à taxa de cura em cinco anos de $96 \%$ em uma série de $50 \operatorname{casos}^{12}$. Entretanto, é desconhecida a repercussão dessa cirurgia sobre a topografia e mobilidade da JUV e da uretra proximal. Cogita-se a hipótese de a cirurgia de MMK-B, comparada às técnicas tradicionais, posicionar melhor a JUV e diminuir a hipermobilidade pós-cirúrgica da JUV. Esta hipótese jamais foi estudada e sua comprovação é necessária para respaldar a recomendação desta cirurgia no tratamento da IUE. A aquisição desses novos conhecimentos poderá contribuir para melhorar o entendimento das alterações determinadas pelas cirurgias propostas para a cura da incontinência urinária de esforço na mulher e obtenção de melhores resultados terapêuticos.

O objetivo deste estudo foi avaliar, por meio da ultrassonografia perineal, a topografia da JUV e da UP nas condições de repouso e esforço físico em mulheres com IUE e identificar as modificações topográficas promovidas pela cirurgia combinada de MMK-B no período pós-operatório.

\section{Métodos}

Trata-se de um estudo de intervenção, longitudinal e prospectivo. Os critérios de inclusão foram: mulheres cuja queixa principal era a perda de urina aos esforços, associada ou não à urgência miccional ou urge-incontinência, cistocele ausente ou presente nos graus I a II, de acordo com a padronização da Sociedade Internacional de Continência ${ }^{13}$ e indicação cirúrgica para correção da IUE. Os critérios de exclusão foram: cirurgia prévia para IUE e gravidez. A amostra foi constituída por 32 mulheres com idade de 32 a 66 anos, média de idade de $48 \pm 8,5$ anos, média de gestações 4,0 $\pm 1,9$, média de partos transpelvianos de 2,8 $\pm 2,1$, média de cesárea de $0,6 \pm 0,9$ e IMC médio de 29 $\pm 4,4$. Destas mulheres, 15 (47\%) estavam na menacma e $17(53 \%)$ na pós-menopausa. Apenas uma paciente (3\%) não apresentou IUE objetivamente demonstrável.

A ultrassonografia perineal da JUV e UP foi realizada com aparelho da marca General Eletric LOGIC 5 após a paciente urinar (bexiga com menos de $50 \mathrm{~mL}$ de urina). A paciente assumia a posição ginecológica e um transdutor vaginal convexo, com frequência de $8 \mathrm{MHZ}$ e revestido com condom estéril, era suavemente colocado entre os grandes lábios, com a pressão necessária para obtenção das imagens, mas sem exercer qualquer compressão que comprometesse o deslocamento das estruturas a serem investigadas. Foram obtidas imagens do pube, uretra, JUV e bexiga com a finalidade de aferir a DPU (distância pubouretral), DVJUV (distância vertical da JUV), DHJUV (distância horizontal da JUV) e UP (comprimento da uretra proximal). As medidas foram efetuadas com base em um sistema ortogonal de coordenadas cartesianas, cujo ponto de origem era a borda inferior da sínfise púbica. O eixo das ordenadas se orientou verticalmente e o das abscissas, horizontalmente à borda inferior do púbis. Estimou-se a distância em milímetros da JUV em relação ao eixo das ordenadas e abscissas, sendo anotados os resultados em números positivos quando acima da linha horizontal, ou negativos quando abaixo. Foram realizadas e registradas no monitor sempre duas medidas, sendo a primeira em repouso e a segunda com a paciente fazendo a manobra de Valsalva, com o propósito de verificar a amplitude do deslocamento das estruturas anatômicas em questão. 
Os seguintes parâmetros foram analisados: - DVJUV: comprimento de uma linha reta longitudinal traçada da borda inferior da sínfise púbica até o encontro de uma reta transversal iniciada na JUV; - DHJUV: comprimento da reta transversal iniciada na JUV até o encontro da linha vertical, com origem na sínfise púbica; - DPU: comprimento de uma linha horizontal traçada da borda inferior da sínfise púbica à uretra (o cruzamento é denominado ponto uretral); - UP: distância da JUV ao ponto uretral. A diferença entre a medida obtida em repouso e a medida obtida no esforço constituiu a mobilidade ou deslocamento $^{7}$ (Figura 1). Era considerada portadora de IUE a paciente com perda de urina pela uretra sincrônica com o esforço físico e ultrassonografia perineal evidenciando deslocamento vertical da JUV maior que $9 \mathrm{~mm}^{7}$.

A cirurgia combinada de MMK-B era realizada com a incisão à Pfannenstiel. No espaço de Retzius, identificava-se a junção uretrovesical, uretra, fáscia do terço inferior da vagina e junção vésico-vaginal. Nas suturas da fáscia pubocervical, foi usado fio prolene zero montado em agulha cilíndrica de $2,5 \mathrm{~cm}$ para colocação de um ponto paralelo à uretra, distando $2 \mathrm{~cm}$ da uretra e $1 \mathrm{~cm}$ abaixo da base vesical, e 1 ponto perpendicular à uretra, lateralmente ao ponto anterior, distando $1 \mathrm{~cm}$ deste e $1 \mathrm{~cm}$ da base vesical. Esses procedimentos foram igualmente realizados do outro lado da uretra. Os fios dos dois pontos mediais eram então fixados na sínfise púbica e amarrados com moderada tensão para elevar a fáscia parauretral (chamados pontos de Marshall). A seguir, os fios dos pontos laterais transfixaram os ligamentos de Cooper bilateralmente (chamados pontos de Burch), mantendo-se uma orientação paralela entre os fios, e eram amarrados com moderada tensão para elevar a fáscia parauretral. Todas as cirurgias foram realizadas pelo primeiro pesquisador.

Os resultados das variáveis contínuas (DPU, UP, DHJUV, DVJUV e seus deslocamentos) foram expressos por suas médias e desvios padrão. Os resultados das variáveis categóricas (IUE e IUU) foram expressos por suas frequências e percentuais. Para a comparação dos resultados no pré e pós-operatório, foi usado o teste $t$ de Student para amostra pareada quando as variáveis cumpriam os critérios do teste de normalidade, e o teste pareado de Wilcoxon quando não cumpriam. Foi adotado o intervalo de confiança de $95 \%$ e o nível de significância considerado quando o valor de $\mathrm{p}$ fosse menor que $0,05(\mathrm{p}<0,05)$.

O estudo foi aprovado pelo Comitê de Ética em Pesquisa em Seres Humanos do Centro de Ciências da Saúde da Universidade Federal de Pernambuco (UFPE). As pacientes foram informadas em linguagem acessível que participariam de uma pesquisa clínica e assinaram o termo de consentimento livre e esclarecido.

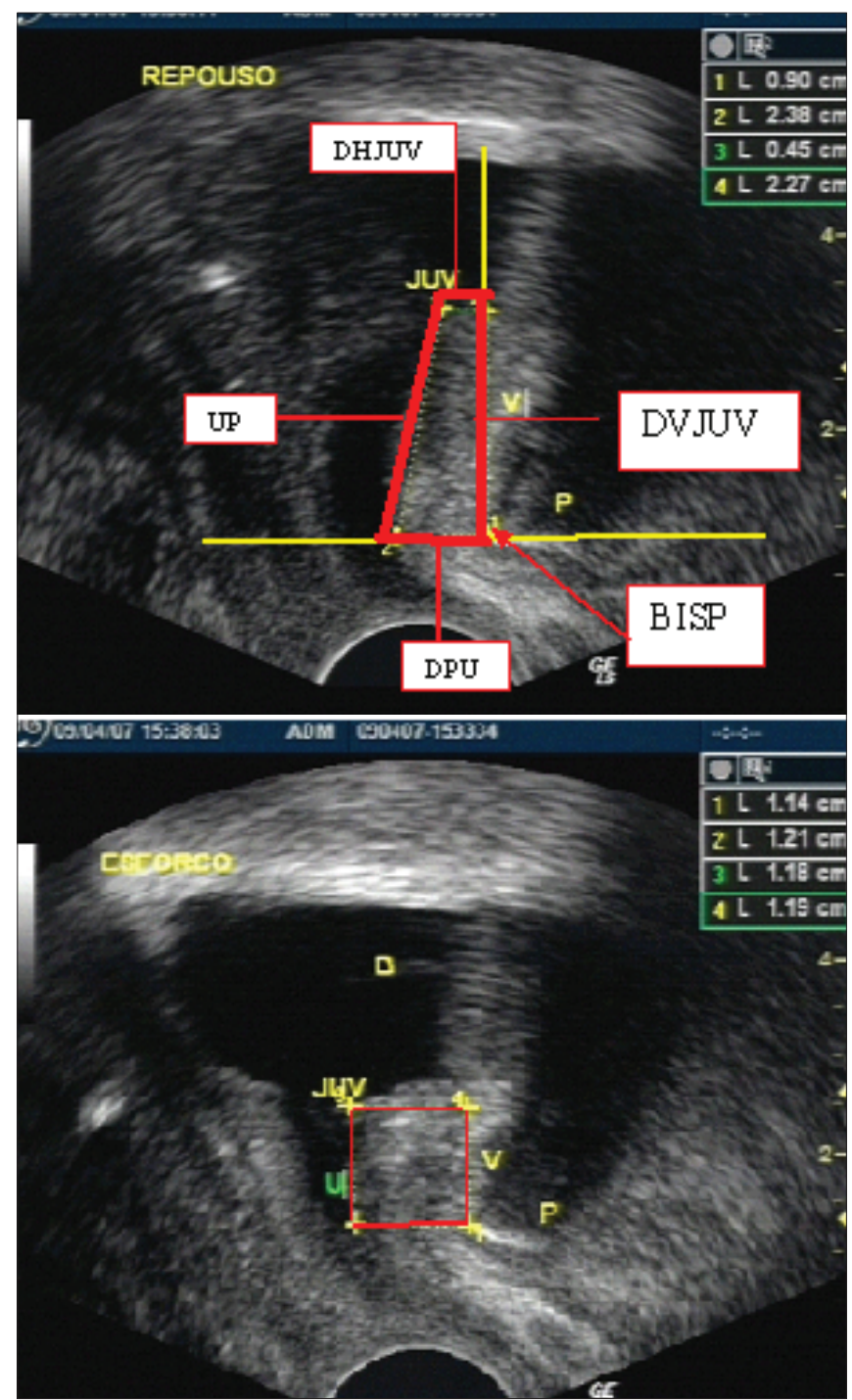

Figura 1 - Imagem ultrassonográfica transvulvar mostrando bexiga, uretra proximal e pube, orientando a forma de obtenção das variáveis DPU, UP, DHJUV e DVJUV. Imagem obtida com a paciente em repouso e em esforço físico.

\section{Resultados}

Nas avaliações realizadas 30 dias após a cirurgia, foi constatado que nenhuma das pacientes apresentava perda urinária aos esforços e uretrocistocele. Das 24 pacientes que apresentavam IUU, 19 delas obtiveram remissão da queixa (79\%).

Os resultados das variáveis contínuas (DPU, UP, DHJUV e DVJUV) e respectivos deslocamentos estão apresentados na Tabela 1.

Cotejando as médias das variáveis ultrassonográficas do pré-operatório com as do pós-operatório, constata-se que a cirurgia de MMK-B produziu alterações topográficas significantes: reduziu discretamente a DPU no repouso $(13,4 \times 10,2 \mathrm{~mm})$ e acentuadamente durante 
Tabela 1 - Distribuição das médias e desvios padrão, em milímetros, das variáveis DPU, DHJUV, UP e DVJU V, e respectivos deslocamentos, obtidos por meio da ultrassonografia transvulvar durante o repouso e esforço nos períodos pré e pós-operatório de mulheres submetidas à cirurgia de MMK-B para correção de IUE

\begin{tabular}{|c|c|c|c|c|c|c|}
\hline \multirow{2}{*}{ Variável } & \multicolumn{2}{|c|}{ Repouso } & \multicolumn{2}{|c|}{ Esforço } & \multicolumn{2}{|c|}{ Deslocumento } \\
\hline & Pré-op & Pós=op & Pré-op & Pós-op & Pré-op & Pós-op \\
\hline DPU & $13,4 \pm 4,3$ & $10,2 \pm 2,5^{\star}$ & $19,8 \pm 5,6$ & $9,0 \pm 2,3^{\star}$ & $6,4 \pm 5,9$ & $-1,2 \pm 2,8^{\star \star}$ \\
\hline UP & $16,7 \pm 5,6$ & $19,7 \pm 4,3^{*}$ & $1,6 \pm 3,2$ & $15,4 \pm 4,6^{\star}$ & $15,1 \pm 4,9$ & $4,3 \pm 3,1^{\star \star}$ \\
\hline DHJUV & $14 \pm 5,9$ & $4,3 \pm 3,1^{*}$ & $20,8 \pm 6,1$ & $6,4 \pm 3,6^{\star}$ & $6,8 \pm 6,3$ & $2,1 \pm 3,1^{\star \star}$ \\
\hline DVJUV & $16,2 \pm 6,4$ & $18,7 \pm 4,7^{\star \star \star \star}$ & $-5,4 \pm 8,9$ & $14,8 \pm 4,6^{*}$ & $21,6 \pm 8$ & $3,9 \pm 3^{\star \star}$ \\
\hline
\end{tabular}

${ }^{*} \mathrm{p}<0,05$ (teste de Wilcoxon para amostras pareadas; ${ }^{* \star} p<0,05$ (teste de $\mathrm{t}$ de Student); ${ }^{* \star *}$ ñ̃o significativa; Pré-op: pré-operatório; Pós-op: pós-operatório.

o esforço físico (19,8 x 9,0 mm). O deslocamento do ponto uretral durante o esforço físico se deu no sentido da sínfise púbica. Reduziu em três vezes a DHJUV no repouso $(14 \times 4,3 \mathrm{~mm})$ e esforço físico $(20,8 \times 6,4 \mathrm{~mm})$, e a mobilidade horizontal da JUV durante a manobra de Valsalva (rotação posterior da JUV) também foi reduzida em três vezes $(6,8 \times 2,1 \mathrm{~mm})$; O comprimento da uretra proximal aumentou discretamente no repouso e acentuadamente no esforço $(1,6 \times 15,4 \mathrm{~mm})$, o que configura um aumento de nove vezes em seu comprimento. Foi observado, também, aumento acentuado DVJUV (-5,4 x $14,8 \mathrm{~mm}$ ) e redução em 5,5 vezes da mobilidade crâniocaudal da JUV (21,6 x 3,9 mm) durante o esforço físico. A cirurgia de MMK-B não alterou significativamente a altura da JUV em repouso.

\section{Discussão}

A avaliação ultrassonográfica pré-operatória identificou importantes alterações anatômicas da JUV e da uretra proximal: discreta rotação posterior da JUV na condição de repouso com significativo agravamento durante o esforço físico; exagerado afastamento uretral do pube durante a manobra de Valsalva; JUV discretamente baixa em repouso e muito baixa em esforço físico, ficando embaixo da musculatura do assoalho pélvico, para fora, portanto, da zona de transmissão da pressão abdominal para a uretra ${ }^{4}$, configurando uma média de mobilidade vertical duas vezes o limite superior de normalidade ${ }^{4,7,8}$. Tais observações sugerem grave lesão das estruturas de suporte uretral, atestando a situação crítica das estruturas de apoio: fáscia pubocervical e músculo pubococcígeo.

No presente estudo, foi observado que a cirurgia de MMK-B promoveu importantes modificações na JUV e uretra proximal. Imprimiu discreta, mas significativa, aproximação da uretra ao pube na condição de repouso e grande impacto na DPU durante o esforço físico, pois impediu o seu afastamento do pube. Quando comparado o deslocamento pré-operatório ao pós-operatório, foi constatado que a cirurgia inverteu esse deslocamento, antes ântero-posterior, tornando-o póstero-anterior no pós-operatório. A mobilidade horizontal da JUV (rotação posterior) foi 3,2 vezes menor após a cirurgia, atingindo valores inferiores aos obtidos pela cirurgia de Burch ${ }^{14}$. Entende-se que com a aproximação do terço inferior da parede anterior da vagina à sínfise púbica, haveria uma redução na transmissão da pressão abdominal para a parede anterior da uretra e aumento da transmissão da pressão para a parede uretral posterior, deslocando a uretra em direção ao pube, como pode ser observado na avaliação ultrassonográfica do pós-operatório. É possível deduzir que, neste nível, a cirurgia de MMK-B reforça o apoio à JUV e uretra proximal, otimiza a transmissão da pressão abdominal para a uretra, corrige a rotação posterior da JUV e impede a hipermobilidade no sentido ântero-posterior.

As médias da DVJUV no pós-operatório $(18,7 \mathrm{~mm}$ no repouso versus $14,8 \mathrm{~mm}$ no esforço e deslocamento de 3,9 $\mathrm{mm}$ ) apontam as importantes e significativas modificações que a cirurgia de MMK-B imprime sobre a JUV e UP e são próximas às promovidas pela cirurgia de Burch, (médias da DVJUV de $21 \mathrm{~mm}$ no repouso, $17 \mathrm{~mm}$ no esforço e deslocamento de $4 \mathrm{~mm})^{14}$. A cirurgia de MMK-B não alterou significativamente a altura da JUV durante o repouso; entretanto, o impacto foi muito significativo durante o esforço, não permitindo a exagerada descida da JUV, reduzindo em 5,5 vezes a sua mobilidade vertical, a qual ficou muito próxima à média de deslocamento apresentado por nulíparas jovens e continentes $(5,3 \mathrm{~mm})^{15}$. Os resultados sugerem que a aplicação e a tensão das suturas respeitaram a topografia da JUV, pois ela permaneceu na altura normal e com mobilidade também normal. Tais achados reforçam a tese de que a perda do apoio uretral e a hipermobilidade da JUV e uretra proximal, com prejuízo da transmissão da pressão abdominal para a uretra proximal, propiciam a IUE ${ }^{4,14,16,17}$. A combinação das cirurgias de MMK e B associam os aspectos positivos das duas técnicas: suspensão e apoio à JUV, além da coaptação da luz uretral, pela convergência das suturas para a sínfise púbica com pontos de MMK, e suspensão da fáscia parauretral para os ligamentos de Cooper, pelos pontos de Burch ${ }^{18}$.

Especial atenção deve ser dada às suturas, pois, se a tensão é baixa, a hipermobilidade da JUV pode persistir com consequente risco de falha cirúrgica ${ }^{9,19}$, e se a tensão é exagerada, há risco de hipercorreção com seus efeitos 
adversos: disfunção miccional, IUU, enterocele e retocele ${ }^{20}$. A tensão exagerada poderá, ainda, promover isquemia nos pontos de fixação e rotura dos tecidos, prejudicando os resultados. Deve-se evitar a aplicação das suturas muito próximas da uretra (menos de $2 \mathrm{~cm}$ ), pois podem levar à compressão e rigidez uretral.

A cirurgia de MMK-B aumentou discretamente o comprimento da UP durante o repouso e impediu o seu encurtamento durante o esforço físico. Como a uretra proximal e a JUV se encontram acima da fáscia pubocervical e intimamente a ela, fixada através da extensão das fibras musculares estriadas do esfíncter uretral ${ }^{21}$, pode-se deduzir que, do ponto de vista anatômico, é o comprometimento da fáscia pubocervical a principal causa da hipermobilidade uretral e, consequentemente, da IUE. Se a fáscia fosse resistente e não prolabasse durante o esforço, a JUV que está acima dela e a ela fixada não se deslocaria excessivamente. Essa fragilidade, entretanto, não aponta para uma lesão exclusiva do terço inferior da fáscia pubocervical. É possível que o dano esteja mais afastado da região periuretral, nas conexões com o arco tendíneo da fáscia pélvica ou na fixação da vagina com os ligamentos útero-sacro e cardinais, corroborando para esta hipótese o encontro frequente de prolapso dos órgãos pélvicos associados à IUE ${ }^{22}$. A JUV se encontra na união dos terços médio e inferior da vagina e, se ela se desloca em demasia, é provável que o nível 2 ou 3 de suspensão vaginal esteja comprometido. Além disso, reforça importância da lesão fascial na gênese da IUE ${ }^{22}$, a interrupção da perda urinária com a aplicação de suturas exclusivamente sobre a fáscia, impedindo o seu deslocamento exagerado e sem nenhuma interferência direta sobre a musculatura do assoalho pélvico. Outro aspecto chama a atenção para a sede da lesão fascial: se a área da fáscia onde as suturas são aplicadas fosse muito fragilizada, provavelmente não teríamos cerca de $80 \%$ das pacientes curadas cinco anos após as colpossuspensões ${ }^{10}$. As recidivas seriam bem mais frequentes e ocorreriam em tempo mais curto. Estas evidências nos levam a inferir que a fragilidade da fáscia pubocervical também ocorre nos dois terços superiores da vagina, em suas conexões com a parede pélvica e, provavelmente, com intensidade ainda maior do que no terço inferior.

Cotejando a média da DHJUV durante o repouso pré-operatório $(14,0 \mathrm{~mm})$ com a média no pós, $(4,3 \mathrm{~mm})$, foi observada importante redução da DHJUV (14,0 mm $\mathrm{x} 4,3 \mathrm{~mm}$ ), constatando-se que a cirurgia promoveu rotação anterior e verticalização da uretra proximal. Essa percepção faz inferir que o deslocamento anterior da JUV e a verticalização da uretra proximal possibilitam menor transmissão da pressão abdominal para a parede anterior da uretra e maior transmissão para a parede posterior, reduzindo a rotação uretral posterior ao esforço e contribuindo para a manutenção da continência. Por este mesmo motivo, menor será a força da pressão abdominal no sentido crânio-caudal e ântero-posterior exercida sobre a uretra, atenuando os efeitos negativos da pressão sobre a JUV e uretra proximal. Esta mudança gera um efeito impeditivo da hipermobilidade uretral e nos faz acreditar que propicia a manutenção da continência e a redução das recidivas.

O tempo de observação é muito curto para avaliar cura clínica da IUE. Não obstante, as repercussões anátomotopográficas da JUV e uretra proximal foram tão marcantes que poderiam explicar o elevado percentual de cura clínica de $96 \%$ dessas pacientes após cinco anos ${ }^{12}$.

Finalmente, com base nos resultados obtidos, pode-se concluir que a cirurgia de MMK-B aumentou significativamente o comprimento da uretra proximal e promoveu significativas alterações topográficas na junção uretrovesical, reduzindo a sua rotação posterior e impedindo a hipermobilidade vertical durante o esforço físico.

\section{Referências}

1. Abrams P, Cardozo L, Fall M, Griffiths D, Rosier P, Ulmsten $U$, et al. The standardisation of terminology of lower urinary tract function: report from the Standardisation Sub-committee of the International Continence Society. Am J Obstet Gynecol. 2002;187(1):116-26.

2. Minassian VA, Stewart WF, Wood GC. Urinary incontinence in women: variation in prevalence estimates and risk factors. Obstet Gynecol. 2008; 111 (2 Pt 1):324-31.

3. Dietz HP, Hansell NK, Grace ME, Eldridge AM, Clarke B, Martin NG. Bladder neck mobility is a heritable trait. BJOG. $2005 ; 112(3): 334-9$.
4. Sendag F, Vidinli H, Kazandi M, Itil IM, Askar N, Vidinli B, et al. Role of perineal sonography in the evaluation of patients with stress urinary incontinence. Aust N Z J Obstet Gynaecol. 2003;43(1):54-7.

5. Dalpiaz $O$, Curti P. Role of perineal ultrasound in the evaluation of urinary stress incontinence and pelvic organ prolapse: a systematic review. Neurourol Urodyn. 2006;25(4):301-6.

6. Lewicky-Gaupp C, Blaivas J, Clark A, McGuire EJ, Schaer G, Tumbarello J, et al. "The cough game": are there characteristic urethrovesical movement patterns associated with stress incontinence? Int Urogynecol J Pelvic Floor Dysfunct. 2009;20(2):171-5. 
7. Brandt FT, Albuquerque CDC, Lorenzato FRB, Lopes DSG, Cunha ASC, Costa RF. A importância da ultra-sonografia transvulvar na avaliação de parâmetros anatômicos relevantes no tratamento de mulheres com incontinência urinária de esforço. Radiol Bras. 2007;40(6):371-6.

8. Virtanen HS, Kiilholma P. Urogynecologic ultrasound is a useful aid in the assessment of female stress urinary incontinence: a prospective study with TVT procedure. Int Urogynecology J Pelvic Floor Dysfunct. 2002;13(4):218-22

9. Viereck V, Pauer HU, Hesse O, Bader W, Tunn R, Lange R, et al. Urethral hypermobility after anti-incontinence surgery - a prognostic indicator? Int Urogynecol J Pelvic Floor Dysfunct. 2006; 17(6):586-92.

10. Glazener $C M$, Cooper K. Anterior vaginal repair for urinary incontinence in women. Cochrane Database Syst Rev. 2007;(4):CD001755.

11. Rogers RG. Clinical practice. Urinary stress incontinence in women. N Engl J Med. 2008;358(10):1029-36.

12. Brandt FT, Oliveira RR, Brandt CD, Coelho AS. Associação das técnicas Marshall-Marchetti-Krantz e Burch no tratamento da incontinência urinária de esforço. J Bras Ginecol. 1990;100(5/6):105-6.

13. Bump RC, Mattiasson A, Bo K, Brubaker LP, Delancey JOL, Klarskov $P$, et al. The standardization of terminology of female pelvic organ prolapse and pelvic floor dysfunction. AmJ Obstet Gynecol. 1996;175(1):10-7.

14. Viereck V, Pauer HU, Bader W, Oppermann M, Hilgers R, GauruderBurmester A, et al. Introital ultrasound of the lower genital tract before and after colposuspension: a 4 -year objective follow-up. Ultrasound Obstet Gynecol. 2004;23(3):277-83.

15. Brandt FT, Albuquerque CD, Lorenzato FR, Amaral FJ. Perineal assessment of urethrovesical junction mobility in young continent females. Int Urogynecol J Pelvic Floor Dysfunct. 2000;1 1(1): 18-22.

16. Cassado J, Pessarrodona A, Tulleuda R, Cabero L, Valls M, Quintana $S$, et al. Introital ultrasonography: a comparison of women with stress incontinence due to urethral hypermobility and continent women. BJU Int. 2006;98(4):822-8.

17. Barbic M, Kralj B. Effect of intra-abdominal position of the bladder neck and stability of its supporting structures on pressure transmission ratio after colposuspension. Int Urogynecol J Pelvic Floor Dysfunct. 2000; 11 (2):97-102.

18. Quadri G, Magatti F, Belloni C, Barisani D, Natale N. MarshallMarchetti-Krantz urethropexy and Burch colposuspension for stress urinary incontinence in women with low pressure and hypermobility of the urethra: early results of a prospective randomized clinical trial. Am J Obstet Gynecol. 1999;181(1):12-8.

19. Sand PK, Winkler H, Blackhurst DW, Culligan PJ. A prospective randomized study comparing modified Burch retropubic urethropexy and suburethral sling for treatment of genuine stress incontinence with low-pressure urethra. Am J Obstet Gynecol. 2000;182(1 Pt 1):30-4.

20. Viereck V, Bader W, Skala C, Gauruder-Burmester A, Emons G, Hilgers $R$, et al. Determination of bladder neck position by intraoperative introital ultrasound in colposuspension: outcome at 6-month followup. Ultrasound Obstet Gynecol. 2004;24(2):186-91.

21. Haderer JM, Pannu HK, Genadry R, Hutchins GM. Controversies in female urethral anatomy and their significance for understanding urinary continence: observations and literature review. Int Urogynecol J Pelvic Floor Dysfunct. 2002;13(4):236-52.

22. Claydon CS. The evaluation of pelvic organ prolapse. J Pelvic Med Surg. 2004; 10(4):173-92. 